Supporting Information for

\title{
Growth Mechanism and Morphology of Hexagonal Boron Nitride
}

Zhuhua Zhang, Yuanyue Liu, Yang Yang and Boris I. Yakobson*

Department of Materials Science and NanoEngineering, Department of Chemistry, Rice

University, Houston, TX 77005, United States
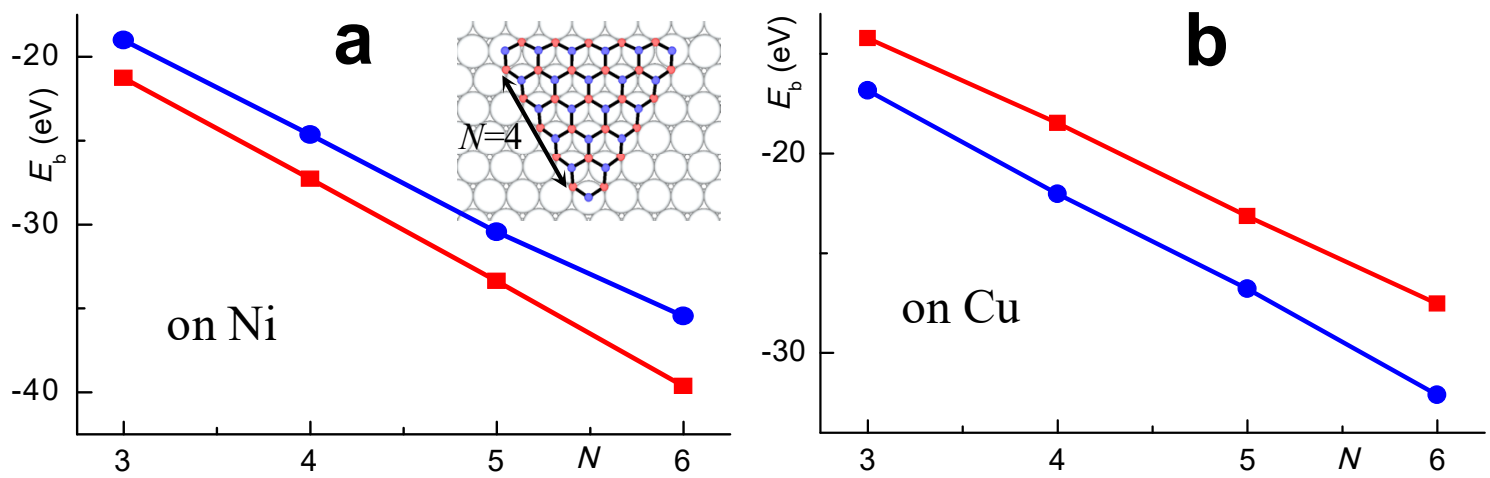

Figure S1. Binding energy of a triangular $\mathrm{BN}$ nanoflake on (a) $\mathrm{Ni}$ and (b) $\mathrm{Cu}$ as a function of flake size $N$. The inset illustrates the atomic configuration of a $\mathrm{BN}$ nanoflake with $N=4$ on $\mathrm{Ni}(111)$. Red and blue lines show the results of B- and N-terminated BN triangles. The slope gives the negative binding energy of the edge on metal. The binding energy of a perfect h-BN monolayer is $2 \mathrm{meV} /$ atom on $\mathrm{Cu}$ and $7 \mathrm{meV} /$ atom on $\mathrm{Ni}$, both being rather weak.
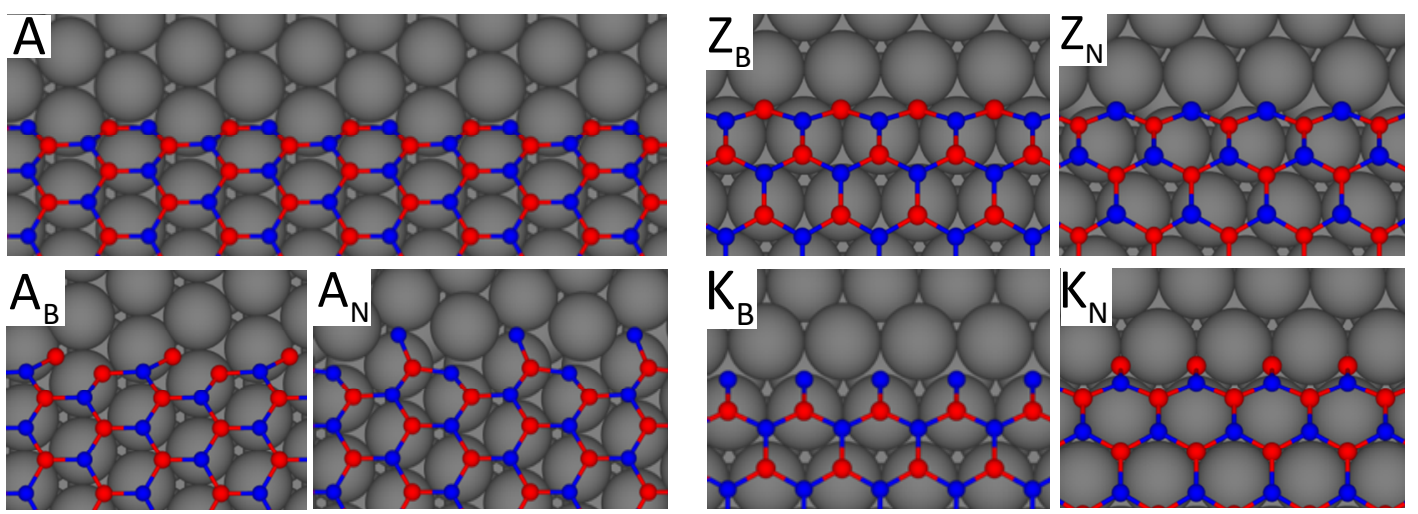

Figure S2. Optimized edge structures of $h$-BN on $\mathrm{Cu}$, including standard armchair (A), B- and $\mathrm{N}$-terminated zigzag $\left(\mathrm{Z}_{\mathrm{B}}\right.$ and $\left.\mathrm{Z}_{\mathrm{N}}\right)$ edges as well as reconstructed ones. 

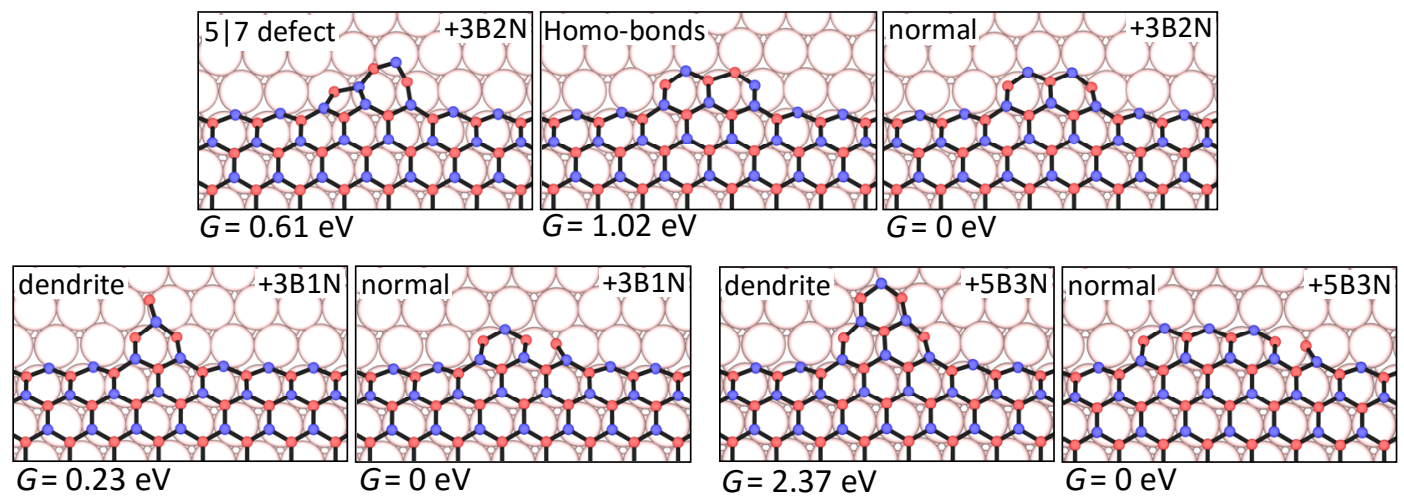

Figure S3. Free energy for the formation of a 5|7 defect, homoelemental bonds, and dendrites of different sizes during the docking of atoms at the $\mathrm{Z}_{\mathrm{B}}$ edge on $\mathrm{Cu}$. The free energies of normally grown structures are set as reference.
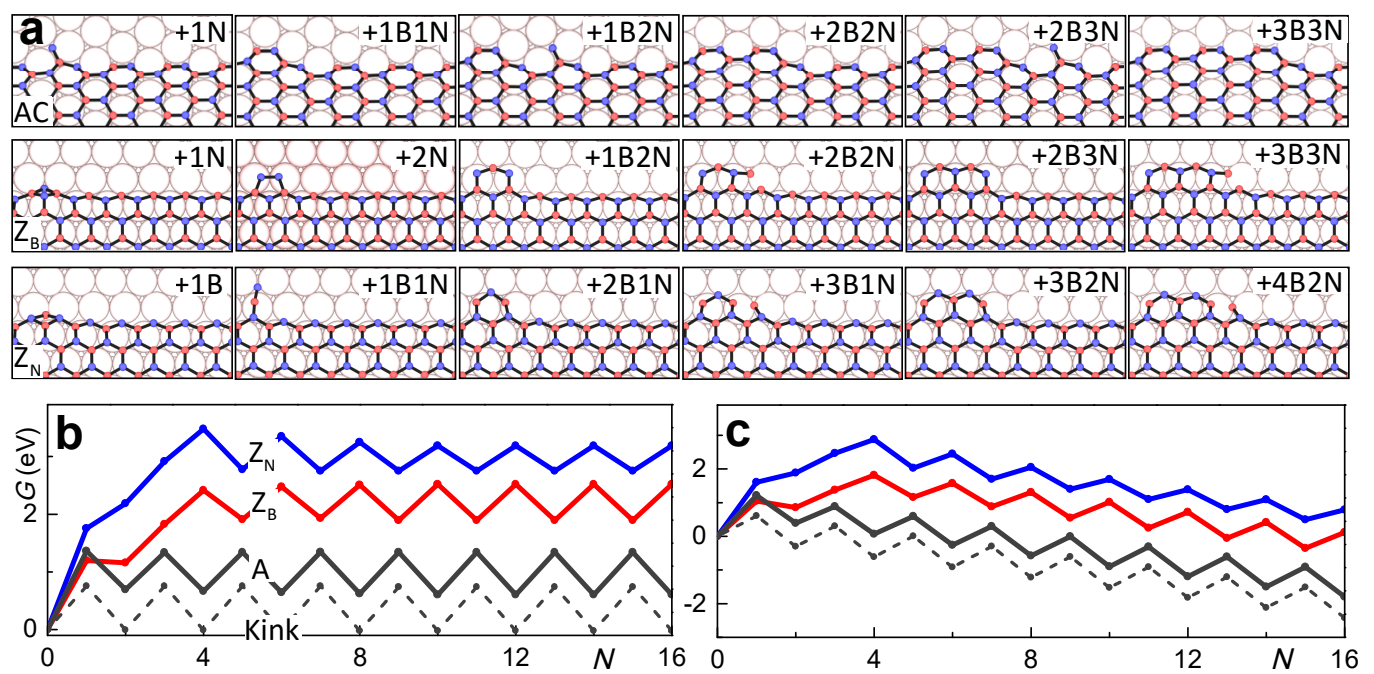

Figure S4. Kinetic models of h-BN growth on $\mathrm{Cu}$. (a) Optimal atomic configurations during the docking of $\mathrm{B}$ and $\mathrm{N}$ monomers to the (top) $\mathrm{A}$, (middle) $\mathrm{Z}_{\mathrm{B}}$ and (bottom) $\mathrm{Z}_{\mathrm{N}}$ edges on $\mathrm{Cu}$. (b) Free energy evolution during the docking of atoms to different edges on $\mathrm{Cu}$ at $\mu_{\mathrm{B}}=0 \mathrm{eV}$ and $\Delta \mu_{\mathrm{BN}}=$ $0.01 \mathrm{eV}$. (c) Free energy evolution during the docking of atoms to different edges on $\mathrm{Cu}$ at $\mu_{\mathrm{B}}=0$ $\mathrm{eV}$ and $\Delta \mu_{\mathrm{BN}}=0.3 \mathrm{eV}$. Dashed line represents the result of kink-flow growth along a skewed $(6,1)$ edge. 

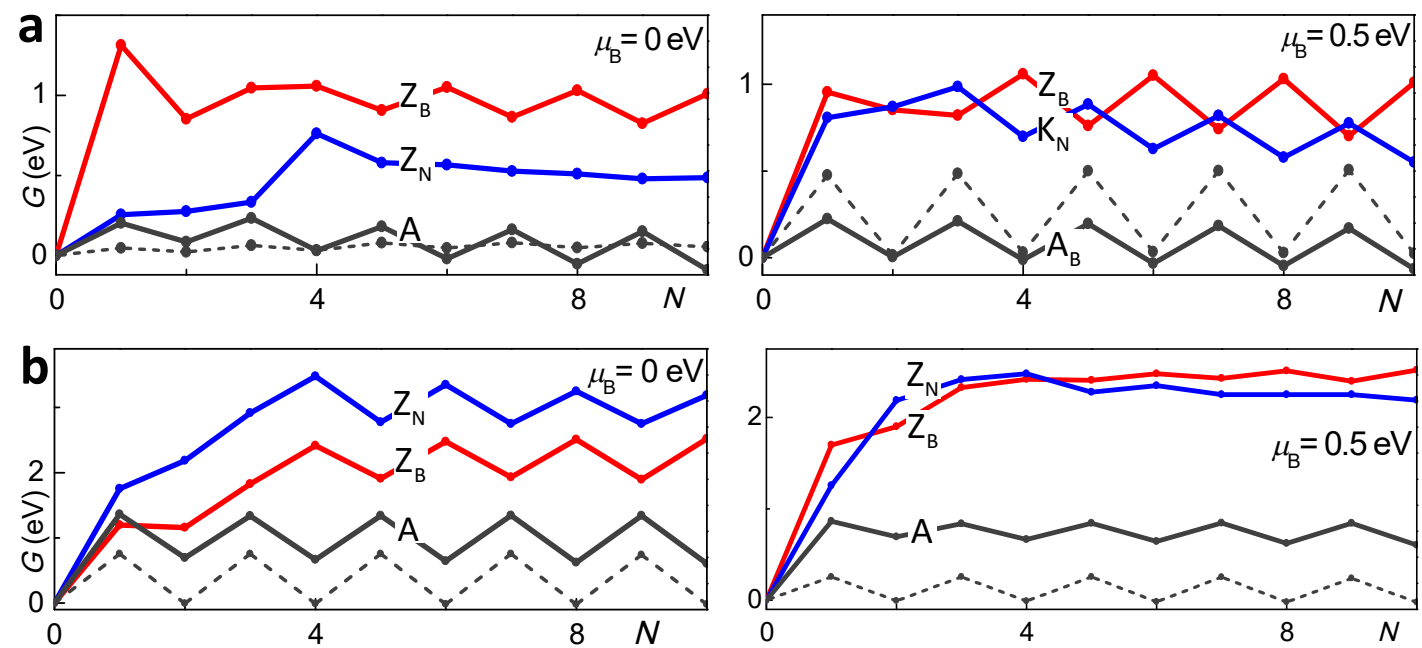

Figure S5. Free energy evolution during the docking of atoms to different edges on (a) Ni and (b) $\mathrm{Cu}$ at different $\mu_{\mathrm{B}}$. Dashed line represents the result of kink-flow growth along a skewed $(6,1)$ edge. $\Delta \mu_{\mathrm{BN}}$ is set to $0.01 \mathrm{eV}$.

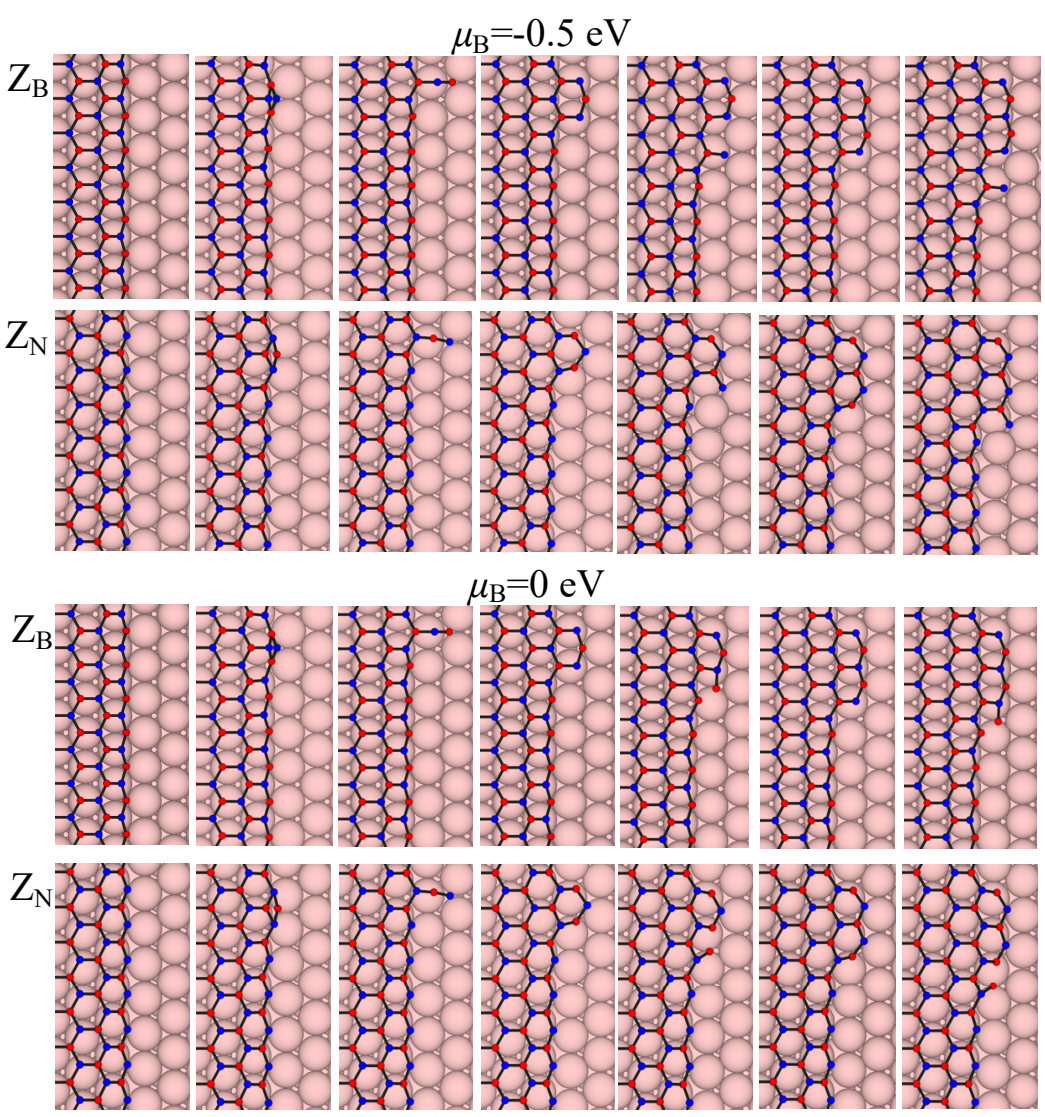

Figure S6. Comparison of optimal atomic configurations during the docking of $\mathrm{B}$ and $\mathrm{N}$ atoms to the $\mathrm{Z}_{\mathrm{B}}$ and $\mathrm{Z}_{\mathrm{N}}$ edges on $\mathrm{Cu}$ at different $\mu_{\mathrm{B}}$. 

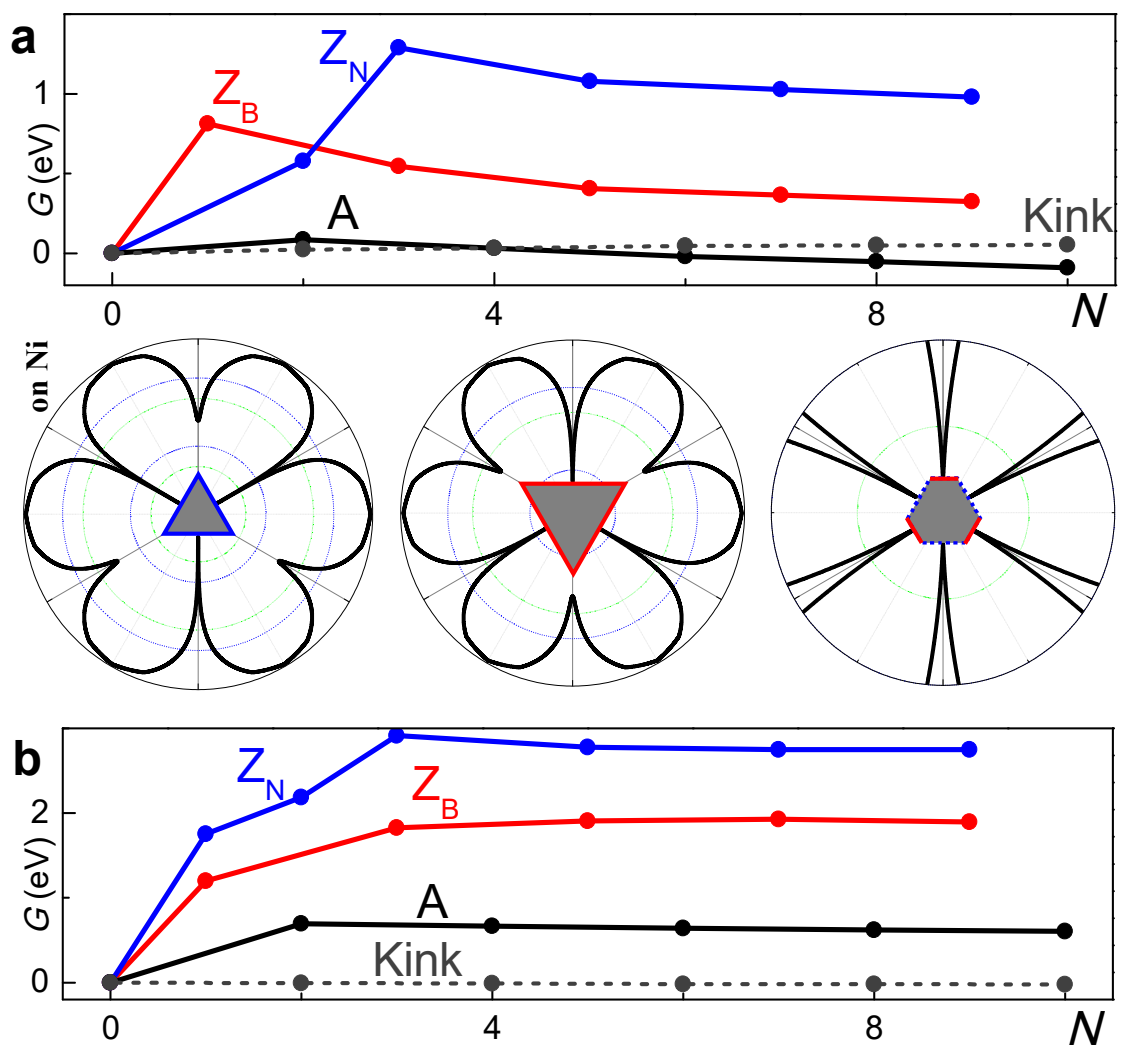

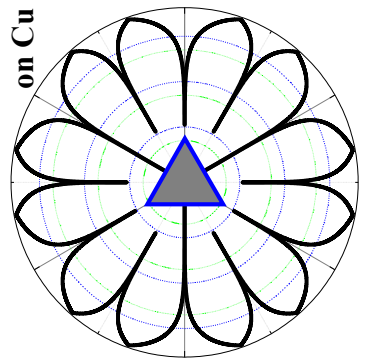

$\mu_{\mathrm{B}}=-0.5 \mathrm{eV}$

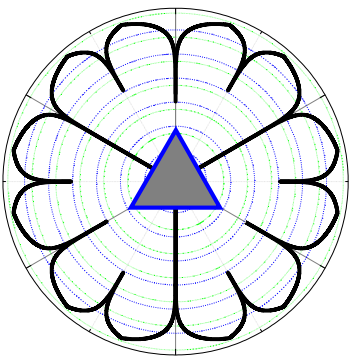

$\mu_{\mathrm{B}}=0.0 \mathrm{eV}$

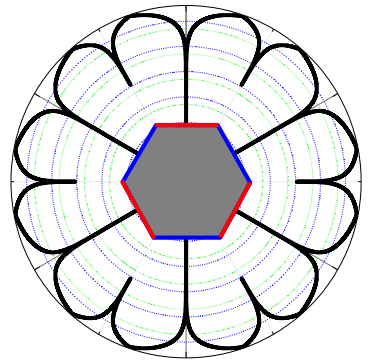

$\mu_{\mathrm{B}}=0.5 \mathrm{eV}$

Figure S7. Kinetic growth of h-BN by feeding mixed monomers and dimers. (a) Top: free energy evolution during the docking of feeding species to different edges on $\mathrm{Ni}$ at $\mu_{\mathrm{B}}=-0.5 \mathrm{eV}$; bottom: kinetic Wulff construction at different $\mu_{\mathrm{B}}$. (b) The same as (a) but on $\mathrm{Cu}$ and at $\mu_{\mathrm{B}}=0 \mathrm{eV}$. Black lines are polar plots of $v(\chi)$ while the red and blue lines show envelope velocities of the $Z_{\mathrm{B}}$ and $\mathrm{Z}_{\mathrm{N}}$ edges (dashed blue for the $\mathrm{K}_{\mathrm{N}}$ edge). $k_{\mathrm{B}} T=0.1 \mathrm{eV}$ and $\Delta \mu_{\mathrm{BN}}=0.01 \mathrm{eV}$. 

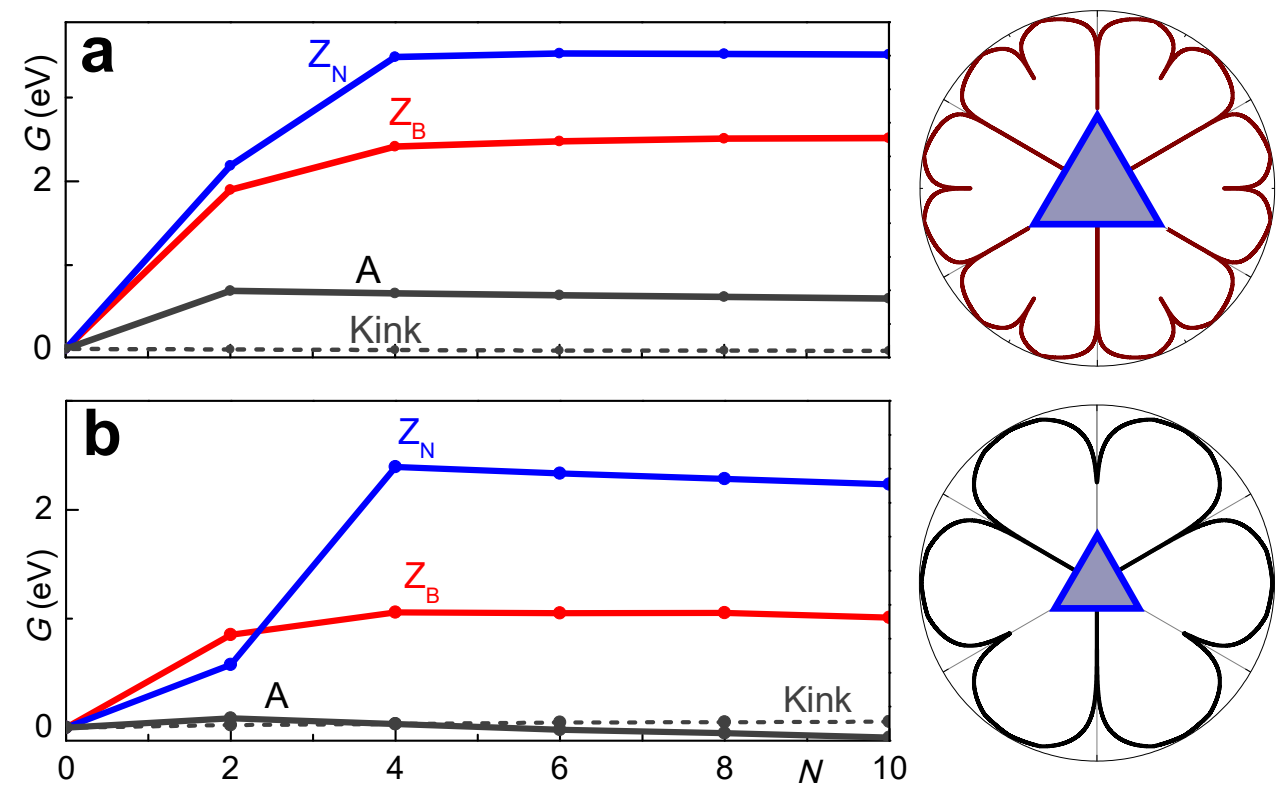

Figure S8. Kinetic growth of $\mathrm{h}-\mathrm{BN}$ by feeding $\mathrm{BN}$ dimers. Free energy evolution during the docking of $\mathrm{BN}$ dimers to different edges on (a) $\mathrm{Cu}$ and (b) $\mathrm{Ni}$, and the corresponding kinetic Wulff construction. $k_{\mathrm{B}} T=0.1 \mathrm{eV}$ and $\Delta \mu_{\mathrm{BN}}=0.01 \mathrm{eV}$.
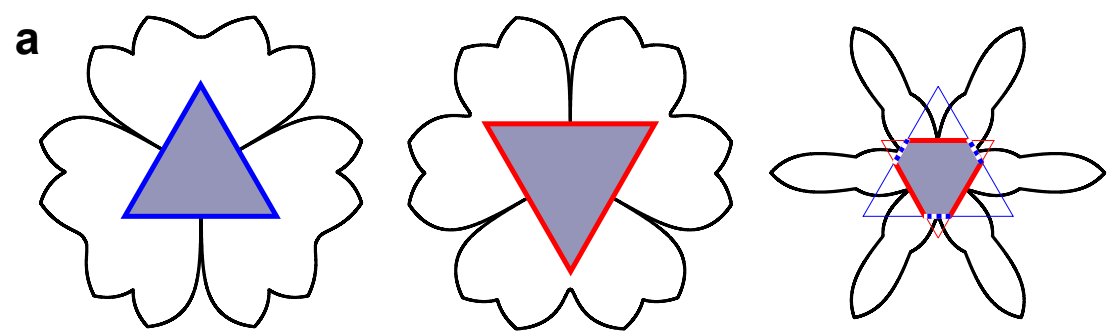

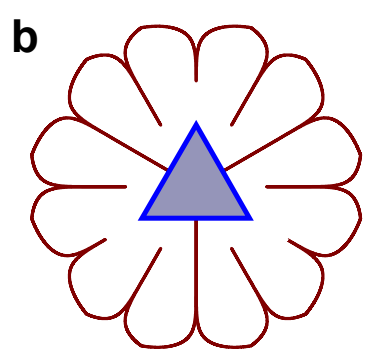

$\mu_{\mathrm{B}}=-0.5 \mathrm{eV}$

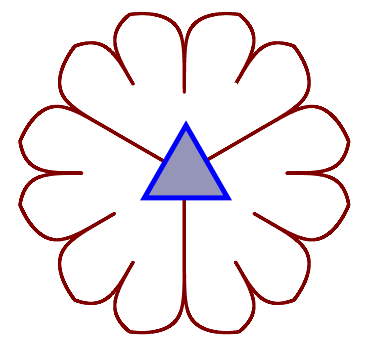

$\mu_{\mathrm{B}}=0.0 \mathrm{eV}$

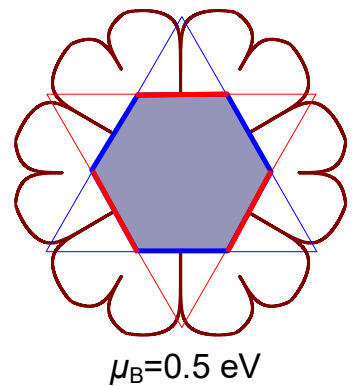

Figure S9. Kinetic Wulff construction for h-BN on (a) $\mathrm{Ni}$ and (b) $\mathrm{Cu}$ at different $\mu_{\mathrm{B}}$. Wine and black lines are polar plots of $v(\chi)$ on $\mathrm{Cu}$ and $\mathrm{Ni}$, respectively, where the red and blue lines show envelope velocities of standard $Z_{B}$ and $Z_{N}$ edges (dashed blue for the $K_{N}$ edge). The plots are in a $\log _{10}$ scale. $k_{\mathrm{B}} T=0.1 \mathrm{eV}$ and $\Delta \mu_{\mathrm{BN}}=0.01 \mathrm{eV}$. 